\title{
Carnets
}

Revue électronique d'études françaises de l'APEF

Deuxième série - 3 | 2015

Insulaire

\section{Les îles lecléziennes : mémoire et initiation}

\section{Bernadette Rey Mimoso-Ruiz}

\section{(2) OpenEdition}

\section{Journals}

Édition électronique

URL : http://journals.openedition.org/carnets/1446

DOI : 10.4000/carnets. 1446

ISSN : 1646-7698

Éditeur

APEF

\section{Référence électronique}

Bernadette Rey Mimoso-Ruiz, «Les îles lecléziennes : mémoire et initiation », Carnets [En ligne], Deuxième série - 3 | 2015, mis en ligne le 28 février 2015, consulté le 30 avril 2019. URL : http:// journals.openedition.org/carnets/1446; DOI : 10.4000/carnets. 1446

Ce document a été généré automatiquement le 30 avril 2019.

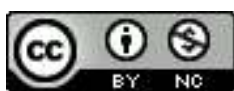

Carnets est mis à disposition selon les termes de la licence Creative Commons - Atribution - Pas d'utilisation commerciale 4.0 International. 


\title{
Les îles lecléziennes : mémoire et initiation
}

\author{
Bernadette Rey Mimoso-Ruiz
}

Le navire glisse sur le miroir de la mémoire Le chercheur d'or

1 Qu'elle soit échappée d'un continent ou surgie des entrailles de la Terre dans le feu d'une éruption, l'île demeure un espace privilégié, résultat des caprices de la nature, peuplée au hasard des migrations. Marquée du double sceau de l'invitation au voyage et de l'enferment : «l'étendue de la mer qui nous tient prisonniers » (Le Clézio, 1985 : 293), elle recèle toujours un mystère pour celui qui l'aborde ou qui l'observe sur une carte. Les mythes, les contes et les légendes, puis la littérature, l'évoquent dès les origines. Il n'est donc pas surprenant que Le Clézio, grand lecteur et voyageur, ait été attiré par ce territoire placé sous le signe du paradoxe, propice à tous les imaginaires et qui, de surcroît, rejoint pour lui la magie de la mer et l'histoire familiale.

Bien que l'œuvre leclézienne soit parcourue d'îles, sans pour autant se résumer à cet espace, nous ne retiendrons ici que celles de l'océan indien : l'île Maurice et ses satellites l'île Plate et l'îlot Gabriel, Rodrigues et la myriade d'atolls qui l'environne, présents dans trois textes qui dessinent une vision originale de l'île pétrie de souvenirs littéraires et de mémoire familiale. Le chercheur d'or et son prolongement Voyage à Rodrigues, premières occurrences significatives de l'inspiration insulaire, évoquent plus directement les grands récits corsaires et les quêtes de trésors enfouis tandis que La quarantaine repose essentiellement sur la saga familiale. Pour autant, après la période que l'on peut qualifier d'« européenne » suivie du « rêve mexicain », le changement significatif du cadre spatial des récits lecléziens correspond au retour sur soi distinctement formulé dans la maturité, dont l'étape de Désert est le premier signe, peut-être impulsé par Jémia venue elle aussi d'un ailleurs et qui inviterait l'écrivain à reconquérir un passé plus ou moins refoulé. Le chercheur d'or représente « une œuvre clé dans l'itinéraire de Le Clézio, initiant une série de romans de nature autobiographique, une «autobiographie » particulière qu'il conviendrait de définir selon le terme avec lequel la critique actuelle désigne ce type de narrations : 'l'autofiction'« (Bernabé-Gil, $2007: 4)$. 
3 Le propos n'est pas d'insister sur ce caractère déjà très largement et pertinemment observé par les critiques et les universitaires, si ce n'est pour noter l'adéquation entre la solitude de l'écrivain (Amar, 2004) et la quarantaine imposée pour des raisons sanitaires aux passagers de l'Ava et l'âge du narrateur Léon $2^{1}$ qui se confond avec celui de Le Clézio lors de son premier voyage à Maurice. Cependant, dans chacun des écrits des repères émergent comme la lente approche d'une quête de soi cherchant à répondre à la lancinante question des origines. En cela, l'île apparaît comme un espace privilégié dans sa double composante d'isolement et de disposition à l'ouverture au monde.

4 Il faut retenir que l'île est l'espace de la solitude par définition et glissement sémantique : isola/île/isolé. Coupée du monde-continent et de son ordre trop rigide car séparé du naturel, l'île est donc le lieu où se conjuguent et s'opposent à la fois liberté et dépendance; y venir ou la quitter oblige à user d'un bateau et dans tous les cas, une préparation s'avère nécessaire. Chacun des récits îliens est précédé d'errances puis d'un long voyage maritime: Alexis vient souvent sur le port de Saint-Louis en quête d'un navire, pris d'un désir d'évasion « Nous étions prisonniers de notre île ( (Le Clézio, 1985 : 102), pour aborder Rodrigues à la suite de jours et de nuits sur le Zeta, après avoir rêvé de quitter Maurice devenu trop étroite. Léon 1, quant à lui, connaît plusieurs escales jusqu'à l'île Plate où se brisent les élans du voyage et ne s'en échappe que pour aller vers un autre espace insulaire, encore plus limité, tandis que ses compagnons se sentent captifs et impuissants. Toutefois, le parcours initial ne constitue qu'une étape, certes préparatrice à la découverte qui suivra, car l'île demeure le lieu par excellence d'un passage initiatique.

Parmi les textes retenus, et même à l'intérieur de chacun d'eux, trois représentations de l'île se croisent et se mêlent pour en faire un espace privilégié, un lieu marqué. Concrètement son évocation repose sur des précisions de géographe et d'historien, comme pour mieux affirmer le réel de cet espace, pour s'écrire ensuite à l'encre des souvenirs de lecture et de l'imaginaire.

\section{Lîle comme entité particulière - L'île comme espace physiquement identifiable}

Dans une mimésis des journaux de bord des navigateurs, les îles lecléziennes sont situées avec une précision scientifique. La présence des cartes, les schémas des constellations, identifient graphiquement les territoires, créent un « effet de réel » tendant à démentir la fiction ou, du moins, à évacuer son caractère chimérique. Le voyage qui sépare Maurice de ses îles satellites comporte de multiples indications sur la route empruntée par le Zeta et pour ce qui est de La quarantaine la présentation de l'île Plate se conjugue autour de coordonnées géographiques qui semblent exclure tout exotisme poétique :

Plate est par $19^{\circ} 52^{\prime}$ de latitude nord, et $57^{\circ} 39^{\prime}$ de longitude est. A environ 20 milles au nord du cap Malheureux, c'est une île presque ronde, dont la forme rappelle, en plus petit, celle de Maurice. (Le Clézio, 1995 : 53).

7 La route maritime se double des chemins stellaires qui guident le timonier plus sûrement qu'un instrument et se lit comme le lien entre le passé et le présent :

Il y a Orion, à bâbord, et vers l'est peut-être le Scorpion où luit Antarès. Celles que je vois avec netteté, en me retournant, à la poupe du navire, si près de l'horizon que je n'ai qu'à baisser les yeux pour les suivre dans leur lent balancement, ce sont les étoiles qui dessinent la Croix du Sud. Je me souviens de la voix de mon père, lorsqu'il nous guidait à travers le jardin obscur, et nous demandait de la 
reconnaître, légère et fugitive, au-dessus de la ligne des collines. (Le Clézio, 1985 :

121).

8 Ainsi la voix du père continue-t-elle de veiller à l'orientation d'Alexis, à prolonger la transmission d'un savoir l'accompagnant de cette manière dans la quête du trésor qu'il n'a pas eu le temps de trouver. En cela, se dessine subrepticement le message profond délivré par l'île Rodrigues et le sens véritable du trésor : comprendre l'ordre du monde, approcher le sens du cosmos. Cartes et plans ne sont finalement que des jalons visibles d'un désir de concrétiser ce qui relève de l'invisible et invite à un ailleurs libératoire, un tour d'horizon allant de l'alpha à l'oméga : le A, d'Alexis initié aux mystères de la vie par le truchement du navire Zeta. L'indispensable médiateur qu'est le bateau est ressenti comme un nouvel Argo plaçant le voyage sous l'égide du mythe de Jason pour annoncer que la quête de l'or dépassera amplement la dimension matérielle au final.

9 L'île se révèle donc espace électif, en raison de son éloignement et des embûches à vaincre avant d'y parvenir, comme une première victoire sur l'adversité.

\section{Lîle, lieu marqué par l'Histoire}

Dans sa représentation de l'île, Le Clézio veille à ne pas séparer les lieux des événements qui les ont traversés afin de relier les paysages aux hommes qui y ont vécu et qui les ont façonnés. En contrepoint, les continents, que ce soit l'Europe ou l'Inde, se font le siège de la violence gratuite. Le chapitre d'une trentaine de pages du Chercheur d'or consacrées à la guerre de 14 est un hapax dans le récit, une parenthèse tragique rappelant les fausses conquêtes qui ne sont que vanité et déshumanisation: "Nous ne nous posons plus de questions, nous n'avons plus le désir de savoir où nous sommes, pourquoi nous sommes là. » (Le Clézio, 1985 : 255). Dans La quarantaine, le récit second de la Yamuna évoque les conséquences humaines de la révolte des cipayes. Le thème de la guerre est constamment présent dans les romans lecléziens depuis Le procès-verbal jusqu'aux derniers écrits et signe l'engagement pacifiste de l'auteur dans lequel se retrouve l'influence de Rabindranâth Tagore ou de Lanza del Vasto.

Représenter l'île signifie aussi dire les violences de l'histoire et les méfaits sur la nature puis sur les habitants, des découvreurs et des colonisateurs. La démarche s'inscrit dans une réalité patente puisque toutes les Mascareignes ne furent habitées que postérieurement à leur découverte. Elles sont, de fait, attachées à une aventure à l'inspiration colonialiste inévitable (Ridon, 2011 : 172) dont l'exotisme est une variante, pouvant se définir comme "le colonialisme de l'imaginaire ", consistant à s'approprier des éléments exogènes pour les remodeler à son gré. La vulnérabilité de l'île a été longuement exploitée par des conquérants européens et les romans se font l'écho de ces irruptions successives. Mais, à partir de ces constatations, Le Clézio s'emploie à dénoncer le système colonial, autre grand thème qui parcourt son œuvre. Ainsi, dans Le chercheur d'or l'immensité des champs de cannes de Maurice, l'organisation des habitations, la hiérarchie sociale qui partage le territoire en zones réservées aux nantis et aux démunis, marquent-elles l'empreinte laissée par l'esclavage avec le souvenir des nègres marrons comme le rappelle Denis dans les promenades dans la forêt à Maurice : «C'était le manger des marrons, avec le grand Sacalavou » (Le Clézio, 1985 : 39) et plus loin, à Rodrigues, dans la bouche d'Ouma : «Mon grand-père était marron, avec tous les Noirs marrons du Morne. Il est mort quand on a écrasé ses jambes dans le moulin à cannes, parce qu'il avait rejoint les gens de Sacalavou » (Le Clézio, 1985 : 228). 
12 Trop souvent, la critique a placé les récits lecléziens dans un espace chimérique et utopique alors que l'approche appartient autant à une réflexion ethnologique et historique qu'à une rêverie autour d'un monde idéalisé. Or, les îles lecléziennes sont vivantes, quasiment humaines, avec leur part de douleurs, d'injustices, mais aussi de beauté.

\section{Les marques de la colonisation}

Les îles de l'archipel des Mascareignes, éparpillées dans les océans par les caprices de la nature apparaissent comme des lieux marqués. Vierges de toute présence humaine, elles concentrent au fil des siècles une population diverse et recréent les structures hiérarchisées des sociétés continentales. Il en va ainsi de Maurice dans Le chercheur d'or où chaque personnage est porteur d'une classe sociale: les mounes que sont les frères L'Etang montrent les deux visages des Blancs installés sur l'île, l'un dévoreur d'hommes et d'espaces (Ludovic) et l'autre plein d'amour et de rêves (le père d'Alexis) qui côtoient les anciens esclaves Captain Cook, et les générations suivantes (son petit-fils, Denis). Sont présents aussi les exclus, coolies relégués dans les champs de cannes, traités à l'identique des esclaves du début du siècle. Rodrigues présente, à une échelle réduite, les mêmes signes d'inégalité et connaît aussi ses marginaux : les manafs, descendants des Indiens tamouls. La quarantaine dresse un tableau similaire dans l'organisation de la communauté d'Européens où Véran s'assimile aux mounes de l'île-mère qu'est Maurice, reproduisant les schémas les plus détestables de la colonisation, tout comme le sirdar tyrannisant les Indiens immigrés est à l'image des surveillants des plantations de cannes. Les trois religions principales de l'océan indien y figurent: les chrétiens, les musulmans et les hindous, comme autant d'espaces cloisonnés. Le Clézio défait l'illusion de l'harmonie des communautés, et en cela, sa vision de l'île s'éloigne de l'utopie qu'on lui accorde généralement ${ }^{2}$. Chacun des récits relate la ségrégation qui prévaut, la puissance de l'argent, le racisme et bien loin d'une vision idyllique, donne à voir un pays morcelé par la volonté des Blancs.

La quarantaine renvoie sur l'île Plate, à l'immigration forcée des coolies après l'abolition et à la permanente organisation sociale dans une hiérarchie destructrice. La présence de l'Inde modifie la représentation de l'île. La Maurice d'Alexis n'est pas celle de Léon car plus imprégnée des esclaves noirs venus de Madagascar, bien que les deux textes se situent dans une période voisine. Le chercheur d'or est plus proche d'une réalité tangible que La quarantaine, qui constitue la vaste métaphore du passage entre Orient et Occident, à la fois dans les liens qui unissent Léon et Suryavati et le biais du récit de la descente de la Yamuna. L'évolution entre les deux romans, parus à dix ans d'écart, reflète celle de l'écrivain qui s'éloigne des romans d'aventure et élargit son approche des mythes originels. Et cette variation fouille plus profondément les secrets de famille. Certes, dans Le chercheur d'or le grand-père Alexis est nommément présent ainsi que les dissensions familiales qui mènent à la ruine, évoquées plus tard encore dans Révolutions, mais $L a$ quarantaine se resserre sur le microcosme de l'île Plate pour, paradoxalement, s'ouvrir davantage au monde. La rencontre avec Suryavati délaisse une symbolique plus simple pour donner la pleine dimension de l'empreinte indienne et hindoue à Maurice. (Poulet, 2006). 
15 L'île ressentie comme berceau des origines, si elle veille à s'appuyer sur des données concrètes n'en demeure pas moins chargée des légendes, des non-dits et des récits familiaux.

\section{Lîle ou l'enfance rêvée}

\section{différentes avec, au centre, l'île Maurice, l'île-mère. En effet, Maurice apparaît comme} l'île achevée, pénétrée d'un ordre qui reproduit, à moindre échelle, les travers des sociétés, mais laisse encore la part de la nature encore vivace dans ses satellites que sont l'âpre et sauvage Rodrigues, les îles perdues de Brandon, de la Frégate et la désolation de l'île Plate et de l'îlot Gabriel. Maurice figure, ainsi que le remarque Kumari Issur, « les îles où s'investit plus particulièrement la faculté créatrice de Le Clézio et où séjournent ses héros. Maurice, Rodrigues, l'île Plate et l'îlot Gabriel sont toutes des composantes du territoire mauricien. »(Issur, $2006: 230$ ). Maurice se fait tantôt espérance d'un nouveau départ dans La quarantaine, tantôt lieu édénique que le sort oblige à quitter pour mieux le reconquérir ensuite dans Le chercheur d'or. Léon, Pierre et Suzanne placent tous leurs espoirs d'une vie nouvelle en direction de Maurice, Alexis ne s'embarque pour Rodrigues que dans l'espérance de trouver le trésor qui lui rendra le jardin de son enfance. Autant de rêves fracassés qui, pourtant, donnent naissance à un renouveau.

Ainsi l'île concentre-t-elle fiction et récit de voyage, mêlant souvenirs personnels et romanesques en écho du voyage baudelairien «pour l'enfant amoureux de cartes et d'estampes/L'univers est égal à son vaste appétit » qui se double des résonances de récits des aventuriers de la mer, navigateurs, pirates, découvreurs, comme autant d'empreintes colorant l'imaginaire ${ }^{3}$. Le Clézio a été bercé par l'image embellie, quasiment mythifiée, d'une île lointaine qu'il a fallu quitter et dont l'absence persiste douloureusement. Michelle Grazier rappelle combien la présence-absence de Maurice a influencé l'écrivain :

Il n'est pas difficile de penser que pour le jeune garçon né au début de la guerre à Nice, entouré de Mauriciens émigrés de la première ou de la deuxième génération, l'île soit une infinie source de rêves, le lieu où, d'une certaine manière, s'enracine l'écriture. (...).

Maurice donc comme une carte postale en couleur dans le contexte des jours sombres de la guerre. Comme un ailleurs lointain où accrocher ses désirs d'évasion. (Grazier, $2010: 22$ ).

En créant l'enfance paradisiaque d'Alexis et de Laure, Le Clézio retrace non seulement le parcours de son grand-père mais exprime la nostalgie de la «patrie perdue » de sa famille et celle d'une enfance qu'il n'a pas vécue. Ces instants d'harmonie des lieux et des hommes reposent sur un certain isolement social, dans un espace que les étrangers perturbent. Les visites sont rares à Boucan, l'oncle Ludovic et son fils dont la condescendance cache mal les intentions de prédateur et le médecin dans les moments graves. Cet équilibre quasi édénique est toutefois illusoire mais l'insouciance de l'enfance n'en perçoit pas immédiatement la fragilité :

Je regarde la grande maison de bois éclairée par le soleil de l'après-midi, avec son toit bleu ou vert, d'une couleur si belle que je m'en souviens aujourd'hui comme de la couleur du ciel de l'aube. (...) Quand j'approche de la maison, j'entends la voix de Mam qui fait réciter des prières à Laure, à l'ombre de la varangue. (...) Je vais jusqu'à la réserve d'eau, derrière l'office, je puise l'eau sombre du bassin avec le broc émaillé. (...) J'entends le bruit doux des oiseaux du soir, je sens l'odeur de la 
fumée qui descend sur le jardin, comme si elle annonçait la nuit qui commence dans les ravins de Mananava. Puis je vais jusqu'à l'arbre de Laure, au bout du jardin, le grand arbre chalta du bien et du mal. Tout ce que je sens, tout ce que je vois alors me semble éternel. Je ne sais pas encore que tout cela va bientôt disparaître. (Le Clézio, 1985 : 23). maternel, renvoie au désir latent d'une compagne féminine à la fois attentive et complice, qu'Ouma concrétisera à Rodrigues. La voix de la mère, cette musique intérieure, restera à jamais attachée au souvenir de ces années dans sa mémoire : «C'est tout ce que je sais d'elle maintenant, c'est tout ce que j'ai gardé d'elle» (Le Clézio, 1985 : 24), tout comme le chemin des étoiles, attaché à la figure paternelle, demeurera son guide.

Si la présence de la mer rejoint les rêveries de l'écrivain sur les rivages méditerranéens, l'océan qui baigne Maurice est fait de fougue et de vigueur : «Poussée par les lames, la pirogue file droit vers le Morne. Le rugissement des vagues sur la barrière de corail est tout proche. Les vagues roulent obliquement, déferlent. » (Le Clézio, 1985 : 51). L'élément marin incarne la séparation d'avec la civilisation consensuelle pour mener vers un ailleurs qui s'éloigne de la doxa pour trouver une vérité naturelle et dont la littérature seule peut exprimer la profondeur.

\section{Lîle creuset des souvenirs littéraires}

21 Hasard des rencontres ou attirance particulières, les îles lecléziennes sont des îles volcaniques et non des fragments de continents arrachés à la terre, elles se placent donc sous le signe du jaillissement et non de la fracture, inscrivant de facto une relation entre l'espace et la mémoire. Surgie du fond des eaux, à l'identique des souvenirs qui remontent aux temps primordiaux, l'île volcanique naît de la rencontre de la terre, du feu, de l'eau et de l'air, dans une synthèse parfaite des quatre éléments. Chacune d'elle est porteuse de secrets oubliés ou enfouis. Lorsque Alexis s'embarque pour Rodrigues avec pour unique bagage une malle et l'image du Corsaire il s'apparente à Jim Hawkins « héritier» de la carte au trésor de Billy Bones, mais la malle d'Alexis n'est pas seulement le contenant de documents conduisant au trésor de Rodrigues, elle matérialise tous les rêves d'enfant et la mémoire du père. Objet quasi fétichisé, elle ne quitte pas le garçon sur le Zeta « m'étant endormi dans la cale, à ma place contre ma cantine » (Le Clézio, 1985 : 126).

\section{Des enfances idéalisées}

Les références littéraires varient d'une île à l'autre, tissant la toile de la fiction, du texte au sens premier du terme ${ }^{4}$. Maurice et Rodrigues s'opposent et sont complémentaires dans Le chercheur d'or car l'une s'attache aux paradis de l'enfance et l'autre est celle des aventuriers. A Maurice plane l'ombre édénique de Paul et Virginie qui sont évoqués à mimot à trois reprises et significativement par des personnes différentes. La première occurrence est le fait de Laure, la sœur tendrement aimée :

Laure me parle aussi de Paul et Virginie, mais c'est une histoire que je n'aime pas, parce que Virginie avait si peur de se déshabiller pour entrer dans la mer. Je trouve cela ridicule, et je dis à Laure que ce n'est sûrement pas une histoire vraie, mais cela la met en colère. Elle dit que je n'y comprends rien. (Le Clézio, 1985 : 64). 
uxième mention se situe vers la fin du roman, alors que la quête du trésor s'est avérée vaine et qu'Alexis est célébré à Maurice comme un héros de la "Grande Guerre ", et cette fois c'est lui qui compare sa sœur à Virginie: «Je lui dis qu'elle ressemble à Virginie et cela la fait sourire » (Le Clézio, 1985 : 282) et la troisième, plus indirecte, alors que Laure s'apprête à rentrer au couvent sonne comme le dernier vibrato d'une époque enfuie : « Oui, un instant encore, son regard brille d'amusement, nous sommes proches, encore, nous sommes " les amoureux », comme disaient les gens autrefois quand ils nous voyaient ensemble. » (Le Clézio, 1985 : 321). Le roman de Bernardin de Saint-Pierre qui a le premier célébré l'île de France renvoie à un passé idyllique détruit par une tempête dont les toponymes expriment la tragédie : "baie du Tombeau, cap Malheureux $»^{5}$ et placent l'île toute entière dans la fusion du réel et de l'imaginaire. La métaphore filée de la maison de Boucan anéantie par le terrible cyclone de 1892 comme un navire échoué renvoie au naufrage du Saint-Géran qui amène la mort sur l'île. Dans Le chercheur d'or, le cyclone ravage la maison mais aussi la famille en réduisant à néant les espoirs du père de sauver sa situation et en les mettant dans l'obligation de quitter la propriété. Comme la mort de Virginie avait conduit Paul au trépas, l'ouragan entraîne la mort du père et précipite la ruine de tous. Toutefois, plus que l'allusion « aux amoureux » qui évoquent les amours enfantines des héros de Saint-Pierre, les liens très particuliers qui unissent Alexis à Laure rappelle ceux existant entre François-René et Lucile dont Chateaubriand se plaît à évoquer le souvenir dans Les Mémoires d'Outre-Tombe (Livre I) :

Lucile était grande et d'une beauté remarquable, mais sérieuse. Son visage pâle était accompagné de longs cheveux noirs; elle attachait souvent au ciel ou promenait autour d'elle des regards pleins de tristesse ou de feu. Sa démarche, sa voix, son sourire, sa physionomie avaient quelque chose de rêveur et de souffrant.

La vie que nous menions à Combourg, ma sœur et moi, augmentait l'exaltation de notre âge et de notre caractère. Notre principal désennui consistait à nous promener côte à côte dans le grand Mail, au printemps sur un tapis de primevères, en automne sur un lit de feuilles séchées, en hiver sur une nappe de neige que brodait la trace des oiseaux, des écureuils et des hermines. Jeunes comme les primevères, tristes comme la feuille séchée, purs comme la neige nouvelle, il y avait harmonie entre nos récréations et nous.

Une complicité identique unit les deux enfants mauriciens :

Laure et moi, nous aimons bien nous asseoir dans une clairière quand les arbres sortent à peine de l'ombre de la nuit, et nous guettons le passage des oiseaux de mer. Quelquefois nous voyons passer un couple de pailles-en-queue. (...) Laure dit qu'ils sont les esprits des marins morts en mer, et des femmes qui attendent leur retour en vain. (Le Clézio, 1985 : 64).

Les liens fraternels évoqués dans Le chercheur d'or semblent préparatoires à la rencontre amoureuse telle qu'elle apparaît dans La quarantaine où Suryavati nomme Léon : «Bahaiii ...Veux-tu être mon frère " (Le Clézio, $1995: 163)^{6}$ ainsi que les liens unissant Alexis à Ouma, double accessible, désirable et chaleureux, à l'opposé de la froideur raisonnée de Laure.

\section{Une robinsonnade à rebours}

De la sœur à la sauvageonne, l'élément féminin est lié à la découverte de l'espace. Des promenades dans le jardin de Boucan aux parcours sur Rodrigues ou l'île Plate chacun des protagonistes figurent des Robinsons survivant dans un milieu qui leur est étranger. Si le 
roman de Defoe résonne inéluctablement dans tout récit relevant de la présence d'un Européen sur une île et s'y adaptant au mieux, la préhension qu'en a Le Clézio diffère du schéma narratif original. En effet, le projet de Defoe relève essentiellement d'une démarche prosélyte auprès des populations indigènes incarnées par Vendredi: les bienfaits de la civilisation apportés au sauvage en même temps que l'expiation d'actions condamnables pour l'Anglais échoué sur une île. De plus, si l'on en croit l'étude de JeanPaul Engélibert consacré au devenir du mythe littéraire de Robinson, l'île des robinsonnades "apparaît comme un espace pur que n'atteignent pas les fléaux de l'humanité. Elle se constitue en territoire où règnent une pureté et un bien absolus, et en tous points, s'oppose au monde d'origine du héros » (Engélibert, $1997: 109)$. Il en va tout autrement chez Le Clézio, en dépit des références nommément présentes, en particulier dans Le chercheur d'or, à commencer par l'absence de naufrage, point de départ obligé des robinsonnades.

Si Robinson, après avoir effectué un inventaire des ressources de lî̂le, parvient au cours des années à allier techniques européennes et opportunités naturelles, Alexis comme Léon sont totalement démunis et ce seront Ouma et Suryavati qui leur apportent les connaissances, non seulement matérielles, mais aussi spirituelles. Pour Le Clézio, le savoir essentiel appartient à l'Autre, à celui qui vit à l'écart de ce qui est nommé la civilisation et ce retour à une forme de primitivisme a souvent été considéré par la critique comme une morale « de boy scout » si l'on en croit Ricardou qui ne reconnaissait à Le Clézio que le mérite de ses tous premiers écrits. Cependant, ces sauvageonnes que seraient Ouma et Suryavati, si l'on s'en tenait aux connaissances livresques, ne possèderaient que des liens étroits avec les éléments naturels alors que vivant à l'écart, elles ont aussi une sagesse forgée par les souffrances qu'elles ont traversées. Ouma et Suryavati sont à la fois filles de l'eau et de la terre. Pêcheuses, elles subviennent à la nourriture de leur famille et en sont responsables : Ouma veille sur son demi-frère Sri, et Suryavati est le soutien de sa mère Ananta. Toutes deux conservent la souplesse et l'agilité des animaux se déplaçant avec autant d'aisance sous l'eau que sur terre : «Elle marche sans bruit, avec des mouvements souples d'animal. » (Le Clézio, 1985 : 188). Femmes de nature, elles portent en elles les éléments vitaux : nourricières et guérisseuses. A la société patriarcale qui régit Maurice, et ses dépendances, Le Clézio oppose un nouvel ordre où l'homme ne devient adulte que par la grâce des femmes qui détiennent les pouvoirs: ce sont elles qui pourvoient en nourriture, elles aussi qui guérissent : Alexis étourdi de soleil doit la vie à Ouma comme une seconde naissance :

Quand je rouvre les yeux, je vois d'abord le feuillage du tamarinier au-dessus de moi, et les ocelles du soleil à travers les feuilles. Je suis couché entre les racines. A côté de moi, il y a un enfant et une jeune fille, aux visages sombres, vêtus de haillons comme les manafs. La jeune fille a un chiffon dans ses mains, qu'elle tord pour laisser tomber des gouttes d'eau sur mes lèvres. (Le Clézio, 1985 : 183).

Quant à Léon, il apprend à vivre sur l'île grâce à Suryavati :

C'est ici que j'ai rencontré Surya, la première fois, et qu'elle m'a soigné, quand je m'étais blessé sur les coraux empoisonnés. Maintenant j'ai appris à marcher sur les récifs, en posant les pieds très lentement, les yeux ouverts dans l'eau transparente. (Le Clézio, 1995 : 135).

Homme-enfant, l'Européen se trouve dans la situation inverse du conquérant et du colonisateur, et ce retour vers la nature n'est pas une régression mais le premier pas vers l'essentiel, le véritable trésor caché dans l'île dont Alexis méconnaissait la teneur : « (...) je n'imaginais pas que cette fille sauvage et étrange connaissait le secret. » (Le Clézio, 1985 : 
296). Quant aux adultes plus conquérants, leur échec est patent ainsi que le note justement Alexandra Jeleva: "dans La quarantaine, les personnages qui tentent de rationnaliser l'espace essuient un échec. » (Jeleva, 2009 : 170).

S'il y a bien rencontre avec l'altérité, elle procède de la reconnaissance et de la métamorphose et se traduit par une relation affective qui relève aussi de la fascination de la double découverte d'une forme de primitivisme et du féminin ${ }^{7}$. La rencontre avec l'Autre sur l'île se traduit par une fusion dans laquelle le sacré est présent. Celui-ci se manifeste de diverses manières, plus symboliques dans Le chercheur d'or, plus explicites dans La quarantaine. En concordance avec la démarche de Le Clézio, le religieux présent à Maurice sous la forme des récits de l'Histoire sainte dont Mam nourrit l'imaginaire de ses enfants, se dissout à Rodrigues et se métamorphose en signes gravés dans la pierre qui dévoilent le secret du monde et l'harmonie avec le cosmos, tout comme l'arbre du bien et du mal qui dominait Boucan se meut en tamarinier porteur de fruits mais qui sera détruit par l'ouragan, tandis que l'arbre chalta demeure blessé mais vivant et seul témoin des années d'enfance. A travers l'image de l'arbre indestructible, c'est l'enfance qui perdure et que l'écriture permet d'immortaliser tandis que le tamarinier, s'inscrit comme l'arbre de la révélation qui peut disparaitre, une fois l'enseignement donné. Le souvenir des mythologies mayas se dessine dans le rapport avec l'arbre chalta qui devient une représentation de l'axe du monde, comme l'enfance reste l'axe central du roman. Les traces laissées sur les pierres renvoient aux marques présentes sur les pierres au Sahara dont il est question dans L'Africain qui sont les signes de la permanence de l'écriture qui doit constamment être déchiffrée.

31 La rencontre avec le sacré est liée avec la rencontre de l'Autre et se sublime dans la relation charnelle tant dans Le chercheur d'or que dans La quarantaine. En cela, l'inscription hindoue est présente qui fait de l'union des corps une plénitude de l'union cosmique pour s'étendre dans $\mathrm{La}$ quarantaine jusqu'au rayonnement. La mythologie transparait également à travers le personnage de Sri, le demi-frère d'Ouma. Le jeune garçon devient une incarnation de Krishna. Désigné par sa mère comme "l'envoyé du Seigneur » il manifeste des dons exceptionnels qui signent son caractère sacré :

Un jour Sri a fabriqué une flûte avec un roseau, et il s'est mis à jouer, tout seul dans la montagne, et c'est ainsi que sa mère a compris qu'il était l'envoyé du Seigneur. C'est lui, quand elle est revenue vivre chez les manafs, qui lui a enseigné à rattraper les cabris à la course, c'est lui qui l'a guidée la première fois jusqu'à la mer, pour pêcher les crabes et les houris. (Le Clézio, $1985: 225$ ).

Toutefois cette influence se métisse d'autres cultures : Ouma porte en elle les stigmates de l'esclavage et a connu une éducation chrétienne lorsqu'elle a été envoyée en France, tandis que la mère de Suryavati est une Anglaise rescapée du massacre de Cawnpore et mariée à un Indien et que la jeune fille rêve de Londres comme d'un paradis inaccessible :

Comment est-ce en France, en Angleterre? Parle-moi de l'Angleterre. Est-ce que c'est très beau avec de grands jardins et des palais, et des enfants qui ressemblent à des princes et des princesses? (Le Clézio, 1995 : 116).

La rencontre avec l'Autre passe par l'écoute: Alexis et Léon sont attentifs aux récits d'Ouma et de Suryavati, à chacun de leurs gestes qu'ils imitent pour mieux s'approcher de leur étrangeté et parvenir à une véritable rencontre. Le silence de l'un et la parole de l'autre tissent les liens et parviennent à une négation de l'altérité : l'un devenant l'Autre (Jarlsbo, 2003 : 168). Si Ouma disparait à la fin du Chercheur d'or, elle reste présente à jamais pour Alexis et Léon quitte tout pour partir vers l'inconnu avec Suryavati. A contrario de Segalen pour qui le Différent demeure énigmatique et renvoie à sa propre 
insuffisance, chez Le Clézio, l'Autre modèle, transforme le moi qui ne sera plus jamais ce qu'il était avant la rencontre.

Les îles lecléziennes procèdent donc de composantes multiples. Territoire propice à toutes les rêveries et ouvert à tous les possibles, elles se dessinent comme le lieu de la rencontre des cultures et des hommes. Profondément marquées par les rêves découvreurs qui se font conquérants et colonisateurs, elles incarnent le meilleur et le pire de l'humanité et se présentent comme des métaphores de l'Histoire. En cela, elles s'éloignent à la fois de la robinsonnade moralisatrice et de l'exotisme lotien pour devenir un espace de métissage, de renaissance et de renouveau laissant percer l'espoir, fût-il fugace, d'une mondialisation fructueuse. Il n'est pas innocent que les romans indianocéaniques soient portés par des adolescents dont l'âge laisse place à l'espoir : leurs dix-sept ans rimbaldiens les situent dans une idéalisation que seul l'espace insulaire peut représenter. Pour autant, réduire le thème insulaire chez Le Clézio à l'océan indien et à une nouvelle écriture de la robinsonnade serait méconnaitre d'autres approches, plus ethnologiques et engagées dans la défense des civilisations menacées, la dénonciation de la disparition des espèces ${ }^{8}$ et l'étude des arts premiers ${ }^{9}$.

\section{BIBLIOGRAPHIE}

LE CLÉZIO, J.-M.G. (1985). Le chercheur d'or. Paris : Gallimard.

LE CLÉZIO, J.-M.G. (1986). Voyage à Rodrigues. Paris : Gallimard.

LE CLÉZIO, J.-M.G. (1987). Haï. Genève : Skira, coll. « Les sentiers de la création ».

LE CLÉZIO, J.-M.G. (1995). La quarantaine. Paris : Gallimard.

LE CLÉZIO, J.-M.G. (1995). Entretiens avec Jean-Louis Ezine. Paris : Arléa.

LE CLÉZIO, J.-M.G. (2006). Raga. Approche du continent invisible. Paris : Seuil, coll. « Peuples de l'eau ».

Le Clézio, J.-M.G. (2011). Les musées sont des mondes. Paris : Gallimard/Musée du Louvre éditions.

***

AMAR, Ruth (2004). Les structures de la solitude dans l'œuvre de J.M.G. Le Clézio. Paris : Publisud.

BERNABE-GIL, María Luisa (2007). La Quarantaine de J.M.G. Le Clézio. Una novela del tiempo". Granada :

Presses universitaires.

BERNABE-GIL, María Luisa (dir.). (avril 2009). Horizons Le cléziens. Toulouse : Institut catholique, Inter-Lignes, numéro spécial.

CAVAllero, Claude (2009). Le Clézio témoin du monde. Clamart : éd. Calliopées.

CAVAllero, Claude (dir.). (2011). Le Clézio, Glissant, Segalen : la quête comme déconstruction de

l'aventure. Chambéry : Université de Savoie.

CAVALLERo, Claude (dir.). (2009). Europe, n 957-958. 
CHAHINE, Béatrice (2010). Le chercheur d'or de J.M.G Le Clézio, problématique du héros. Paris : L'Harmattan.

DUTTON, Jacqueline (2003). Le chercheur d'or et d'ailleurs. L'Utopie de J.M.G. Le Clézio. Paris :

L'Harmattan.

ENGELIBERT, Jean-Paul (1997). La postérité de Robinson Crusoé. Un mythe littéraire de la modernité.

Genève : Droz.

GRAZIER, Michèle (2010). « Paysages de Maurice », in Thierry Léger, Isabelle Roussel-Gillet, Marina Salles (dir.). Le Clézio, passeur des arts et des cultures. Rennes : Presses universitaires, coll.

«Interférences », pp. 21-28.

ISSUR, Kumari (2006). « Les îles indianocéaniques de Le Clézio », in Bernadette Rey Mimoso-Ruiz (dir.). J.M.G. Le Clézio. Ailleurs et origines : parcours poétiques. Toulouse : Editions universitaires du Sud, pp. 227-235.

JARLSBO, Jeana (2003). Ecriture et altérité dans trois romans de J.M.G. Le Clézio. Désert, Onitsha, La quarantaine. Lunds Universitet.

JELEVA, Alexandra (2009). «L'aventure de Léon dans La quarantaine », in Maria-Luisa Bernabé-Gil (dir.). Horizons Le cléziens, Actes du colloque de Grenade, Inter-Lignes numéro spécial, Toulouse, Institut catholique de Toulouse, pp. 169-177.

JOLLON-BERTOCCHI, Sophie, THIBAUlt, Bruno (dir.). (2004). J.M.G. Le Clézio. Lectures d'une œuvre. Nantes : Éd. Du Temps.

LEGER, Thierry (2010). «Le Clézio, passeur des arts et des cultures », in Thierry Leger, Isabelle Roussel-Gillet, Marina Salles (dir.). Le Clézio, passeur des arts et des cultures Rennes : Presses universitaires, coll. «Interférences ».

LEGUAT, François (1934). Voyage et aventures de François Leguat en deux îles désertes (1690-1698), publiés par Jacques Bouenger. Paris : Plon.

NATUREL, Mireille (2003). « L'alchimie intertextuelle dans Le chercheur d'or de Le Clézio », in B. Degott, P. Nobel. Images du Mythe, Images du Moi. Mélanges Offerts à Marie Miguet-Ollag Nier, Presses universitaires de Franche-Comté, pp. 39-52.

onimus, Jean (2006). Pour lire Le Clézio. Paris : Presses universitaires de France, coll. « Ecritures ». POULET, Régis (2006). « L'Inde et Le Clézio : héritages et choix », in Bernadette Rey Mimoso-Ruiz (dir.). J.M.G. Le Clézio : Ailleurs et origines : parcours poétiques. Toulouse : Editions universitaires du Sud, pp. 219-226.

RAUVille (de), Hervé (1889). L'île de France légendaire. Port-Louis : Challamel \& Cie.

REY MIMOSO-RUIZ, Bernadette (2006). (dir.).J.M.G. Le Clézio. Ailleurs et origines : parcours poétiques. Toulouse : Editions universitaires du Sud.

RIDON, Jean-Xavier (2011). « Segalen, Le Clézio, Glissant et l'ambiguïté insulaire », in C. Cavallero (dir.). Le Clézio, Glissant, Segalen : la quête comme déconstruction de l'aventure. Chambéry : Université de Savoie, pp. 171-183.

ROUSSEL-GILLET, Isabelle (2011). J.M.G. Le Clézio écrivain de l'incertitude. Paris : Ellipses.

THIBAult, Bruno (2009). J.M.G. Le Clézio et la métaphore exotique. Amsterdam : Rodopi.

THIBAUlt, Bruno, MOSER, Keith (dir.). (2012). J.M.G. Le Clézio. Dans la forêt des paradoxes. Paris :

L'Harmattan. 
SALLES, Marina (2006). Le Clézio. Notre contemporain. Rennes : Presses universitaires.

SUZUKI, Mazao (2007). J.M.G. Le Clézio : évolution spirituelle et littéraire. Par-delà l'Occident moderne.

Paris : L'Harmattan.

\section{NOTES}

1. Nous désignons les deux Léon par Léon 1 et Léon 2, en concordance avec le temps du récit.

2. A noter toutefois les mentions faites dans Le chercheur d'or de la république du Libertalia qui fascine Alexis. Cf. Daniel Defoe, Libertalia : une utopie pirate [1724-1728], traduction de Guillaume Villeneuve, Paris, l'Esprit frappeur, 1998 http://fr.wikipedia.org/wiki/Daniel_Defoe.

3. A deux reprises le nom de François Leguat est cité. Ce récit de voyage Les naufragés de Dieu : aventures d'un protestant et de ses compagnons exilés en deux îles désertes de l'océan Indien, 1690-1698 / Voyage et avantures de Francois Leguat et de ses compagnons en deux isles desertes des Indes orientales, ...de Leguat, François, 1708. Notre référence de lecture est Voyages et aventures de François Leguat en deux îles désertes (1690-1698). Texte revu et publié par Jacques Boulenger, Paris, Plon, 1934.

4. Etymologiquement « texte » signifie « tissu».

5. Ces lieux sont situés au nord de l'île, face à l'île Plate.

6. Une autre occurrence apparaît vers la fin du roman : «Elle se tourne vers moi, elle dit mon nom Bahii, Mera Bahii » (Le Clézio, 1995 : 362).

7. Ce primitivisme est lié aux récurrences textuelles "c'est la première fois " dont Isabelle Roussel-Gillet fait une analyse pertinente dans son ouvrage J.M.G. Le Clézio écrivain de l'incertitude, Paris, Ellipses, 2011, aux pages 50 à 53.

8. Pour exemple Pawana, Paris, Gallimard, 1992, est consacré à la chasse aux baleines. La mention du massacre des tortues dans Le chercheur d'or en est aussi une illustration.

9. Cf. Haï, Genève, Skira, coll. « Les sentiers de la création », 1971 (rééd. Champs Flammarion, 1987), Raga. Approche d'un continent invisible, Paris, Seuil, coll. «Peuples de l'eau », 2006 et Les musées sont des mondes, Paris, Gallimard/Musée du Louvre éditions, 2011.

\section{RÉSUMÉS}

L'espace privilégié que constitue l'île appartient aux paysages lecléziens dont les deux romans Le Chercheur d'or et La quarantaine expriment l'essence pour en donner la portée symbolique. Liées à l'histoire de sa famille mais aussi attachées aux lectures d'enfance et aux rêves d'aventure, les îles lecléziennes sont porteuses d'une poésie faite du paradoxe où l'évasion vers les horizons marins le dispute au sentiment d'être prisonnier d'une terre isolée. Cet article se donne pour objectif l'étude des îles lecléziennes dans leur dimension sociohistorique où se retrouvent les composantes de la colonisation, mais aussi l'évocation d'une enfance réinventée. L'espace insulaire des Mascareignes, sous des couleurs parfois utopiques, prend la dimension d'un territoire propice à la rencontre avec l'altérité et à l'initiation au sacré par l'entremise de l'insulaire, renversant les codes établis dans les romans traditionnels.

The privileged space that an island represents is an integral part of the landscapes described by Le Clézio whose two novels Le Chercheur d'or and La quarantaine express the very essence of that 
space in order to convey its symbolic significance. Connected to his family history and also linked to the books he read in his childhood and to his dreams of adventure, the islands in the works of Le Clézio possess a poetry which express the paradox where evasion towards the horizon of the open seas appears to be in opposition with the feeling of being a prisoner in an isolated spot. The aim of this article is to study Le Clézio's islands in their socio-historic dimension where all the aspects of colonisation are to be found as well as the evocation of a reinvented childhood. The setting of the Mascarene Islands, painted in colours that are sometimes utopian, takes on the dimension of a territory conducive to a meeting with alterity and to an initiation into the sacred through the influence of insularity, thus upsetting the codes established in traditional novels.

INDEX

Mots-clés : altérité, enfance, île, Le Clézio (J.-M. G.), poétique de l'espace

Keywords : alterity, childhood, island, Le Clézio (J.-M. G.), the poetic aspect of space

\section{AUTEUR}

\section{BERNADETTE REY MIMOSO-RUIZ}

Institut catholique de Toulouse

bernadette.reymr[at]wanadoo.fr 